\title{
Le narratoème, modèle de la nomadanse dans La réfugiée d'Hédi Bouraoui
}

\section{Suzanne CROSTA, Université McMaster}

La réfugiée fait partie de l'œuvre plurielle, multiforme et innovatrice d'Hédi Bouraoui : dix-sept œuvres poétiques, treize romans, cinq contes, neufs études littéraires et essais théoriques et deux narratoèmes. Au cœur de son immense aventure culturelle et projet d'écriture, c'est le cœur de l'humanité que l'on sent battre dans ses mots, entre les lignes et dans ses traversées et ses pratiques artistiques. Le projet d'écriture de Bouraoui est en perpétuel mouvement, axé sur la remise en question des certitudes, l'écoute des souffrances et la recherche d'un langage capable de modifier les conditions de l'expression et de renouveler notre rapport au monde. En effet, communication et communion émanent de la même source aimante. Qui pourrait même penser à distinguer le cœur du Petit prince de celui de Saint-Exupéry: il y a là comme une correspondance, « une médiatisation primordiale » de l'amour et du silence qui nous émeut et nous appelle à la quintessence de l'humanisme.

La réfugiée aborde un sujet brûlant, celui des seuils de tolérance pour les exilés et les immigrants dans les sociétés d'accueil. Le récit retrace le parcours d'une réfugiée laotienne, au nom de DorBoa (surnommée Lotus), en quête constante d'un espace qui l'accueillerait à bras ouverts et qui lui permettrait de vivre une vie décente. Sa trajectoire s'inscrit dans une mouvance contemporaine qui s'interroge sur l'avenir de l'humanisme du vingt-et-unième siècle, d'autant plus que ses expériences de réfugiée soulignent le fait que les seuils de tolérance rétrécissent et que les principes de l'art de vivre n'ont plus cours à l'heure actuelle. Les flux migratoires s'observent sur les îles, les archipels et sur tous les continents, obligeant à une intégration plus problématique que rapide et heureuse. Si les émigrants se perçoivent eux-mêmes comme des évadés d'un territoire où ils ne peuvent ou ne veulent plus vivre, il se peut qu'ils soient vus comme envahisseurs, c'est-à-dire comme une sorte de charge imposée dans la société d'accueil. Fragile et dangereuse perception. Rien de plus opposé à l'esprit de Bouraoui : ni évasion ni invasion, mais respect dans le regard en arrière et le regard sur soi, respect dans le regard en avant et le regard sur l'autre. Ce regard est manifestement l'essentiel du message du grand poète, l'idée directrice de son œuvre abondante, et également originale, elle qui manifeste la sincérité d'une vie ou d'une insertion au monde assumées en profondeur. 
La réfugiée poursuit la réflexion déjà amorcée dans Bangkok Blues en créant un espace plus significatif à la rencontre des diversités culturelles, celles de l'Asie, de l'Afrique et de l'Europe (la France) en vue de favoriser un dialogue pris sur le vif, pourvu d'une intériorité à illuminer et à partager. Déjà, sur la page de couverture, s'annoncent trois éléments de lecture : le titre, le sous-titre et l'étrange mot «narratoème ». Le titre est personnalisé par un buste fleuri de jeune fille au visage candide, avec de grands yeux à l'orientale, pleins de clarté tout autant que de mystère : il marque déjà l'orientation du récit et l'on est immédiatement en sympathie. Le sous-titre "Lotus au pays du Lys » suggère et prépare les notions de transplantation et de transvivance, tandis que l'étoile florale révèle son propre sens métaphorique. Enfin, la classification du récit comme «narratoème »- néologisme pour « désigner un poème narratif et un récit poétique »- précise la volonté de transcréer, de traverser les frontières de tout ordre : du savoir, des arts, du vivre-ensemble.

À la lumière de ces constats, nous proposons dans cet article de suivre la trajectoire de la «réfugiée », d'en cerner les regards, les discours et les métaphores qui la concernent. En examinant le vécu de DorBoa/Lotus et les réflexions qu'il soulève, on s'attardera sur les questions et les thèmes rattachés à la nomadiVivance chez Bouraoui. On passera ensuite à l'analyse de leurs rapports avec les composantes et les procédés rhétoriques du narratoème afin de souligner la recherche d'une expression pouvant traduire les pensées intérieures et les expériences vécues des réfugiés, des exilés, des errants, bref, des altérités multiples sous le prisme de la nomadanse. Finalement, on dégagera les traits saillants de la poétique de la relation et du divers, symbolisée par cet hymne à l'AmiAmour ${ }^{1}$, dans ce court récit devenu un long et généreux poème.

\section{Traversées et regards pluriels sur La réfugiée (Lotus au pays du Lys)}

Cette histoire, à penchant allégorique, est celle de DorBoa qu'on suit de sa naissance à sa vieillesse, à travers ses expériences et ses états affectifs. Au départ, on apprend que le pays de son enfance est celui du bonheur et du confort. Au pays du Lotus, sa famille vit «dans les plantes-bandes royales », sous le protectorat de la France, et elle jouit de certains pouvoirs économiques et politiques. Son père Séthiy, un horticulteur, est un haut-commis de l'État, un véritable «gouverneur de la rosée $»^{2}(15)$, alors que sa mère Thempaa (surnommée Frangipane) est une grande dame au niveau national. Mais cette phase paradisiaque de son enfance est de 
courte durée : née la cinquième de la famille, Lotus perd sa mère, ses deux sœurs et son frère. Alors que DorBoa et sa sœur Cactus doivent quitter la maison familiale après le remariage de leur père avec Dorku Lap (surnommée la nouvelle rose), le sort de sa meilleure amie, Dor Eung (surnommée Orchidée) est aussi triste. Suite à l'échec de son mariage arrangé, Dor Eung assume la responsabilité entière de ses enfants dans la solitude et l'inquiétude. Voilà l'effet d'un mariage arrangé qui est à l'image du pays où l'abdication du roi et l'abandon de la France laissent la voie libre au nouveau régime communiste sous l'« œil de Moscou... contrôlant tout » (28).

$\mathrm{Au}$ niveau du quotidien, DorBoa souffre l'humiliation auprès des agents du nouveau régime en raison de ses liens avec la famille royale. «Figée telle une poupée russe » (28), DorBoa voit ses horizons d'avenir s'effriter au gré des slogans et des édits de la propagande du nouveau régime «aux mains communistes » (27). Ayant perdu ses droits d'expression, sa liberté de vivre et de circuler à sa guise, DorBoa quitte son travail à la poste, les siens et ses amis, s'échappe de son pays par forêts et cours d'eau, pour échouer dans les camps de l'Organisation des Nations Unies en pays Thaï. Ces camps de transit où «les réfugiés ne mangent jamais à leur faim » (32) sont un véritable enfer. L'expérience de DorBoa ne sera pas inutile : malgré son lot de souffrances et de frustrations, elle symbolise l'espoir d'un paradigme nouveau, d'une résilience ou résurgence pour les réfugiés en transit, en attente d'un avenir dans un pays d'accueil. Vu sa connaissance du français ${ }^{3}$, DorBoa s'installe en France, tandis que « ses cousines optent pour l'Aigle américain / Les faisant rêver de richesses brillantines... » (35).

\section{a) Heurts et malheurs au pays d'accueil}

Loin des camps et dans l'attente d'un statut légitime au pays lyssois, DorBoa passe son temps à s'occuper des démunis, et cet esprit de bénévolat déterminera son mode de vie en France. Ayant connu le chômage, les emplois de serveuse et de domestique, DorBoa déclare sans ambages : "Quel choc de sa vie d'être descendue si bas ! / Gâtée comme une reine dans son pays / Elle est simple domestique chez les Lyssois » (37). Le sort de cette réfugiée évoque celui qui se dessine un peu partout dans le monde. Mais, une heureuse rencontre! Jasmin, un tunisien originaire de l'île de Djerba, («l'île des Lotophages de la fameuse Odyssée », 42), deviendra son ami fidèle pour le reste de la vie. ${ }^{4}$ Le dialogue ouvert avec ce compagnon révèle la démarche conciliatrice des différences religieuses entre eux. Si Jasmin apprécie la foi «à la loupe laïque », sous l'angle rationnel et œcuménique, il en est tout autre pour DorBoa qui s'investit particulièrement dans le bouddhisme depuis sa tendre enfance. Elle affiche les signes extérieurs 
de sa foi, collier et médaille, et elle entretient l'autel où se trouve la statue en bronze du Bouddha. Détachée du matériel, elle mène une vie ascétique, observant une discipline tant du corps que de l'esprit, et elle entretient une relation pacifique avec son entourage. Elle suit et vit avec simplicité la foi bouddhique sans vraiment en maîtriser intellectuellement les préceptes et les détails historiques $(69 ; 91)$.

Cette acceptation des différences approfondit leur amitié. À son compagnon devenu confident, elle s'ouvre et parle des défis qu'elle rencontre en France. D'abord, et pendant une décennie, la possibilité d'obtenir un emploi, à temps plein et à la hauteur de ses compétences, ne s'est jamais réalisée. Pour survivre, elle se résigne à des emplois à temps partiel, à faible rémunération, sans avantages sociaux et dont la dure réalité finit par la pousser vers une retraite précoce, provoquée par la maladie et le chômage (78-79). L'impossibilité de se réaliser pleinement accuse ses sentiments de marginalisation, cherchant refuge dans la vision nostalgique du pays d'origine. Elle souhaiterait y retourner, mais ironiquement son statut de réfugiée lui bloque cette voie :

Avec la carte de réfugiée...

Je pouvais circuler partout

Sauf au Laos, Thaïlande, Vietnam

Il me fallait devenir Lyssoise pour revenir au pays natal. (79)

Il n'y a pas que cette nostalgie : DorBoa tient à garder à tout prix son identité laotienne, même en sacrifiant ses chances d'obtenir la nationalité lyssoise. Chez elle, il n'y a pas de compromis vers la double identité : elle est et demeure Laotienne même en sol lyssois. Elle y vivra par contrainte, sans vraiment en épouser les valeurs, étant soucieuse des traditions laotiennes et bouddhistes, qu'elle transmettra sous forme de conte ou d'histoire à qui voudra l'entendre (Phra Bang de Luang Prabang, dame qui pouvait incarner bouddha, 70-71; l'odyssée de la princesse laotienne Savivanh Sang Manivong, 102-105).

\section{b) La marginalisation de l'altérité}

Cette relation pénible avec « le pays du Lys » suscite la réflexion sur le seuil de tolérance de la France envers ses communautés culturelles diverses. Au fil des vers, des strophes et des chapitres exposant l'aventure humaine du couple, se dressent en contrepoint les défis multiples que rencontrent les réfugiés: le froid, la nourriture nouvelle, les pratiques xénophobes d'ostracisme et d'exclusion, même dans les espaces publics (83-84). Ainsi, un Lyssois ne veut 
pas partager avec le couple un jardin public (84) parce qu'à ses yeux, ce sont des intrus. Quoique ce soit un baume de penser à la terre ancestrale et d'être fidèle à ses traditions culturelles, cela ne purifie pas l'atmosphère empoisonnée (84-85).

Le narrateur procède ainsi à élargir le débat en poursuivant les considérations politiques rattachées aux pratiques discriminatoires de la majorité. Outre le cas de la réfugiée, on relève des stéréotypes concernant les Asiatiques, lesquels sont accusés de trafiquer les cartes d'identité de leurs confrères et consœurs décédés en France pour faciliter l'entrée en France d'autres Asiatiques (85) :

Au pays du Lys, on dit :

«Les Asiatiques ne meurent jamais ! »

Quand un des leurs décède... sa carte d'identité

Servira à un autre clandestin

Un nouveau destin au citoyen avec papier (85).

Cette reprise des rumeurs dans un langage direct, où on reproduit les invectives du « on » « au pays du Lys », attire l'attention du lecteur sur le poids du discours de la majorité sur les communautés minoritaires. Et la condition des nouvelles générations asiatiques et maghrébines installées dans les banlieues ne s'étant pas améliorée, elles agissent d'une façon gênante, avec colère, en révolte: «Des jeunes sans vergogne bousculant / Raison cartésienne / La malmenant... la triturant dans les banlieues...»(86) Le narrateur se solidarise avec les personnages, exprime leurs frustrations et interroge une si injuste discrimination dans le domaine de l'emploi, comme celle que le cousin de Jasmin a subie. Le récit oppose les rumeurs et les préjugés du « on » au cas particulier d'un jeune qui a bien voulu suivre tous les stages mais qui, malgré tous ses efforts, n'arrive toujours pas à trouver d'emploi (87). L'emploi des trois points en suspension troue la fluidité de l'expression et souligne la frustration du petit cousin vis-à-vis d'une situation sans issue :

Petit cousin de Jasmin...

Fleur de grenadier a suivi tant

De stage de croissance...

Sans obtenir la moindre broutille !

Son nom à pépins durs à avaler l'exclut...

...des gorges fertiles

Sa réinsertion aux pétales floraux laisse à désirer

Il a beau tailler les encornures...

... s'ajuster au modèle des justes causes....

Rien n'y fait... Il restera toujours 
L’épine au flanc des fleurs... (87)

Le narrateur s'inquiète de la marginalisation économique des jeunes, laquelle les rend facilement vulnérables à la criminalité car, pour le narrateur, la solution n'est pas de leur côté : «Mais qui trouvera le remède miracle / Qui pourrait calmer ces révoltés (86) ». Comme le montrent les cas de DorBoa, de Jasmin et des jeunes gens asiatiques et maghrébins, les organisations opèrent en circuit fermé, alors que le drame se joue dans la vie quotidienne de la nation. Chez Bouraoui, ce n'est même plus la dialectique singulier/pluriel, ni particulier/général, ni individu/communauté : c'est une nette négation de l'idée même de bien commun et, par le fait même, le refus d'un humanisme épanouissant qui est au cœur des préoccupations du récit.

\section{c) La tolérance répressive}

Ce constat amènera le narrateur à poser son regard accusateur sur le leadership politique. Dans ses considérations spontanées sur les petitesses des grands partis politiques de France, Jasmin n'a pas de misère à ironiser. La rose (PS, parti socialiste), c'est le parti des paresseux, dont la préoccupation première est l'obtention du fauteuil présidentiel, en quoi les sans-papiers pourraient aider ! Pour leur part, les pétales bleus (UMP, union pour un mouvement populaire), c'est le parti des travailleurs. Il est bien facile d'ajouter: dans l'un ou l'autre cas, on travaille pour les partis. Les douteux sparages de VerboZéro, de Beurette Amère et d'autres tontons politiques lyssois n'ont été que des coups d'épée dans l'eau: peut-être leur manquait-il l'étiquette républicaine ! Lotus n'est guère impressionnée et demeure silencieuse, réfugiée dans sa foi : sa sérénité autant que sa sincérité finissent par rallier son compagnon pour répéter avec elle une apaisante prière bouddhique, pas plus expressive qu'un mantra pour lui.

Tandis que les démunis n'ont que la religion et leurs rêves comme refuge, la famille royale bénéficie de la générosité du pays hôte, comme on le voit dans le cas de Savivang :

Savivang habitait dans... ...une maison offerte par l'État

Femme au foyer...

Elle touchait une allocation familiale

Elle n'était riche que du titre... ...de réfugiée...comme nous!

Son mari pharmacien s'est intégré superbement ! (103)

On dirait, suivant une réflexion entendue maintes fois, que l'eau va à la rivière. Dans la réalité brutale, La Fontaine ne s'est pas trompé : «La raison du plus fort est toujours la meilleure » (Fables, L. I, 10 : «Le Loup et l'Agneau »). Comme le suggère le récit de Bouraoui, la réussite 
et la protection des réfugiés dépendent de leurs capacités de répondre aux attentes des lois de la jungle du pays d'accueil. «Au pays du Lys», il faut de l'argent, ou un titre, ou une recommandation importante pour s'implanter favorablement, et nos deux amis, DorBoa et Jasmin, en sont dépourvus.

\section{d) NomadiVivance}

Cet état de fait aura pour conséquence inévitable de séparer DorBoa et Jasmin et de redéfinir leurs relations interpersonnelles. Celui-ci quittera le pays qui n'a pas su l'accueillir, et tentera sa chance dans le pays de l'érable, tandis que DorBoa fera un court séjour en Afrique («République caroubière », 117) pour rendre visite à sa nièce et resserrer les liens avec sa famille étendue. Cette nomadiVivance, au miroir des valeurs et des aspirations de nos deux personnages, s'avère une quête de liberté dans les flux et reflux de leur vie en relation avec l'autre, au-delà des frontières géopolitiques. À titre d'exemple, lors de son voyage en Afrique, DorBoa baignera dans une atmosphère culturelle et religieuse vraiment différente, surtout dans le rapport avec l'Islam : l'impressionneront particulièrement le sacrifice («égorgement») de huit moutons pour fêter la naissance de la petite rose et la fête d'Aïd El Kébir. Malgré l'hospitalité africaine basée sur le principe donnant-donnant et la grande générosité de ses hôtes, DorBoa se rend compte que nonobstant la bienveillance de sa parenté en Afrique, les différences culturelles sont trop grandes pour qu'elle puisse s'y installer et elle rentre « au pays du Lys ».

Sa réflexion est à la mesure de sa pérégrination: elle n'avait souhaité qu'une implantation en pays du Lys, mais c'était devenu un long périple. Maintenant, avec tous les arômes qu'a humés son âme ardente, mais en oubliant tant et trop d'épines, elle pourrait répéter, après le poète Paul Claudel : «Comprends cette parole à l'oreille de ton âme qui ne résonne que parce qu'elle a cessé » (124). Du « pré-carré d'Asie » (122) dont elle n'avait jamais oublié ni renié les parfums, elle est passée par le jardin des différences dont elle ne pourra oublier les fragrances étonnantes. Maintenant chez elle, au plus intime d'elle-même, elle rejoint les mêmes enthousiasmes, car elle a fait l'unité en elle-même. Elle porte résolument, en son foyer intérieur, une mosaïque ou peut-être un arc-en-ciel, en tout cas un monde en couleurs par lequel elle aime les humains dans ce qu'ils ont d'humanité. Des fois, elle se demande comment elle a été entraînée dans ce long itinéraire de grâce. 


\section{Le narratoème : poésie et prose en images}

Le récit intime de DorBoa évoque de par ses images décousues non seulement la fragilité de sa condition de réfugiée, mais à la fois les sources jaillissantes de sa nomadiVivance, car «elle dit la Vivance » (NomadiVivance, 13 ; l'auteur souligne). L'écrivain s'est ainsi creusé un nouveau puits, d'eau ou de lumière, comme on voudra. Il peut tout autant abreuver ou éclairer. Et il a donné un nom à ce type de puits : le narratoème, un néologisme désignant le poème narratif ou le récit poétique, en fin de compte le mariage noologique de la narration et du poème, d'une réalité concrète et d'un idéal, d'une vie exubérante et d'un appel intérieur, qui n'exclut pas le mysticisme. Considérer différemment les genres selon une perspective critique et une démarche transcréatrice permet de déchiffrer la polyphonie des parcours culturels et littéraires de $L a$ réfugiée.

\section{a) L'expression et les composantes du narratoème}

Dans 1'«Avant-propos », l'auteur signale les trois éléments clés du narratoème: le transvasement de genres, l'interpénétration de formes et la traversée de contenus culturels des plus variés (9). L'œuvre bouraouienne s'affranchit de définitions génériques fixes et joue souvent sur le croisement des genres, les béances interstitielles, les pratiques transartistiques afin de mieux traduire, exprimer, traverser « les interstices des pays, des continents, des cultures, des civilisations, des philosophies... Ceci pour ne privilégier aucune composante dans son unicité inaltérable et d'éviter toute hégémonie d'une culture unique » (10).

En tant que prose, le texte de Bouraoui n'épouse pas, évidemment, la structure particulière qui caractérise la poésie. Il n'embrasse pas non plus les conventions formelles imposant la structure d'une prose classique. Il adopte plutôt une démarche strophique entrelaçant la narration à la troisième personne, des dialogues $(62 ; 64 ; 66)$ et de la grammaire implicite, inexprimée $(41 ; 62-63 ; 125)$. Au niveau de la langue, Bouraoui se livre à un jeu d'homophonie. Il déploie la rime poétique en reprenant un ou quelques phonèmes. Par exemple : une rime en [for] dans les vers : «Pourvoyeur...forts/À contrôler...coffres-forts » (15); une rime en [fã] dans les vers : «Au pays...d'éléphants/Tirant...les enfants »(15). Les signifiants de tels phonèmes se rapprochent sur le plan phonologique, mais les signifiés ne sont pas identiques. En faisant rimer certains phonèmes et pas d'autres, l'auteur fait un choix délibéré lui permettant d'attirer l'attention sur les mots de son choix, de sa fantaisie. Claudel ne l'aurait pas désavoué, lui qui 
écrivait dans Cent phrases pour éventails : «Il faut qu'il y ait dans le poème un nombre tel qu'il empêche de compter » (109).

\section{b) Le narratoème : modèle par excellence de la nomadanse}

Outre la recherche d'un nouveau langage, de jeux de sonorités, de rimes et de rythmes troués ou saccadés, l'enjeu du narratoème et de l'œuvre repose sur cette altérité interculturelle qui permettrait d'ouvrir de nouvelles perspectives sur le savoir et la sensibilité des sujets pluriels. Dans les parcours de DorBoa (laotienne) et de Jasmin (tunisien), la nomadanse s'épanouit dans « la danse artistique, esthétique de vivre le pluriel cadence de l'être et de l'Avoir, des éléments de la Terre et du Ciel, de toutes les contingences dans l'univers de l'actuel » (nomadiVivance, 13). Cette nomadanse trouve son expression ici dans le narratoème dont les jeux et les enjeux du roman/poème, du poème narratif, du récit poétique suggèrent tantôt la césure, la béance, l'amalgame ou l'inversion. Contrairement à la définition que donne Georges Chapouthier du genre - «[... l'aboutissement en mosaïque de la combinaison et de l'intégration de diverses formes littéraires : prose et poésie s'y mêlent, dans un écoulement lyrique ininterrompu de versets harmonieux, propres à dessiner les tesselles du réel et du rêve » (20) - le narratoème chez Bouraoui accorde plus d'importance à l'interstice, à la béance, aux figures de styles, en particulier, à la métaphore, à la métonymie et à l'allégorie, pour sonder la profondeur de la conscience dans le monde. La définition de Nafée Nelly Faïgou abonde dans ce sens : «le narratoème devient alors une tour de Babel reconstituée avec les moyens de bord: de la ponctuation, du pollen, la complicité infaillible des abeilles, et du lecteur. Un nectar que chacun hume et déguste à volonté »(182). Il faudrait également ajouter que, dans La réfugiée, le narratoème accueille la parole, le silence, la cadence de la nature et des cultures, les contraires réunis et les sensibilités diverses. Il se mue en mosaïques de chœurs, d'écrits, de tableaux, d'images. Dans la dynamique des croisements de genres, de formes et de thèmes variés, l'imaginaire fait appel à un véritable réseau sensible où tous les sens sont sollicités : le visuel, l'olfactif, le toucher, l'ouïe et le goûter. Privilégiant le flou, le clair/obscur, La réfugiée s'ouvre à la beauté de nouvelles métaphores florales, aux plaisirs de divers parfums, à la délicatesse ou à la fermeté de ses composantes. C'est plein d'expressions langagières nouvelles, et l'on y retrouve des huiles et des épices qui relèvent les plats culinaires de diverses cultures. 


\section{c) Le narratoème : du réel au déploiement analogique du sens}

Chez Bouraoui, il n'est pas question de synthèse des genres, mais d'une recherche ou d'une tendance formelle qui libère sens et expressions, avec les potentialités fécondes qui émergent de la relation avec l'Autre. Ainsi dit-il :

Ouverture, liberté, créations hors normes. L'effet désiré dans ce texte, c'est de prendre plaisir aux permutations des fleurs devenant personnages et vice-versa, de temporalité bigarrée sans démarcation définitive, de changements de lieux, de registres, du réel à l'imaginaire, au légendaire.... Aucune limite dans cette transculturalité et ses transferts constants d'une culture à une autre, etc... (11)

Cette œuvre est remplie d'une foule d'images de la nature. Que de fleurs avec leurs parfums, dans tant de jardins où elles s'épanouissent! Les personnages doublés d'une identité florale déstabilisent une lecture au premier degré et obligent la reconnaissance d'une opacité à découvrir et à interpréter. La flore est omniprésente, mais elle n'incarne point de stéréotypes sexistes, car l'auteur l'applique aux genres, aux partis politiques, aux pays. Dans La parole mutante, Bouraoui fait la lumière sur les sources de sa conscience créatrice :

[...] nous habitons aujourd'hui un «Village global» qui réduit l'espace des communications où tout se transmet simultanément et en un temps record, et d'autre part produit la prolifération d'entités nationales ou fondamentalistes (toutes les religions ont leur propre mouvement) qui rétrécissent les lieux du discours et censurent la différence ! Entre ces deux tensions extrêmes se situe la parole poétique dans la liberté totale et sans prétention aucune de convertir qui que ce soit. Et est là explorant les espaces inconnus et infinis pour nous faire miroiter les enjeux de notre fin de siècle. (13)

Enfin, l'auteur souligne l'impératif de percer les différentes gangues métaphoriques qui figent les identités et les relations entre peuples, cultures, pays... pour rendre la possibilité d'une dérive métonymique («pays du frangipanier » (30), «Révolution lyssoise » (35), « Merdaillaise » (88), «Verbophage » (89), etc.) et en saisir la signification profonde.

La transplantation de Lotus/la réfugiée « au pays du Lys » et son amitié avec Jasmin/ l'immigré né en Afrique, de passage «au pays du Lys » mais ayant «choisi l'érable comme... pays d'adoption» (41), servent d'occasion privilégiée pour que s'ouvrent les cœurs et que s'établisse un dialogue interculturel sur leurs expériences vécues dans le creuset français et, par extension, européen. Il n'y a pas de jeu de séduction ni d'expression sexuelle, mais une recherche de communication et de communion. À travers ses images et sa prose/poésie, $L a$ réfugiée constitue un lieu de confluence des cultures africaine, asiatique et européenne. Le texte de Bouraoui est orné de différents types d'images : dessin - encre de Chine (13); dessin simple 
$(23 ; 67)$; dessin - crayon sur papier (39) ; tableau - acrylique (93), pour ne citer que ceux-là. ${ }^{5}$ Non seulement ces illustrations artistiques embellissent-elles le texte, mais elles assument quelques autres fonctions. Certaines images permettent à l'auteur de planter le décor du segment textuel auquel elles se rapportent $(39 ; 81)$ et, grâce à elles, l'auteur caractérise les personnages faisant l'objet d'un passage précis $(13 ; 23)$. D'autres offrent au lecteur une possibilité de prolonger ou d'imaginer la suite de la trame du récit. Ce n'est donc pas exagéré de dire que les rapports du narratoème et des images se complexifient et deviennent à leur tour un espace signifiant à explorer, un lieu d'observations croisées qui s'ouvrent sur des schémas et des expressions culturelles nouvelles.

\section{d) Le narratoème comme poésie pertinente}

La réfugiée puise dans l'imagerie florale pour souligner la beauté mais également la fragilité de ceux et celles dont le destin les amène à vivre ailleurs (la nomadiVivance). Au niveau de la forme, le narratoème fait le pont entre perspective critique et démarche créatrice, entre roman et poème, entre être et autrui, en déstabilisant les frontières de la pensée, en transgressant les limites du genre et en doublant tout personnage d'une texture florale ou d'un néologisme musical mono-pluri-lingue. Tout au long de cette analyse, on a relevé les surnoms floraux des personnages (DorBoa/Lotus, Thempaa/Frangipane, Dor Eung/Orchidée, etc.), des bribes de leur vie sous couvert végétal, des pensées et des réflexions en métaphores florales, avec les parfums spécifiques. Si le récit joue sur les néologismes avec une certaine verve satirique, c'est surtout quand il frôle les régimes politiques, les réseaux de communication, les modes de vie, les croyances religieuses ou les valeurs contemporaines, tous sujets qui occasionnent de graves impasses à la modernité. Les leaders politiques, à l'exemple de VerboZero et d'Amère Beurette, dont leur plan Big-Bangliou a pour but de « pourchasser les Anti-glandouilles » (87), abusent de leur pouvoir, négligent les dangers liés à l'effervescence sociale qu'entraîne l'emboîtement des langues, des religions et des cultures.

Alors que les surnoms des personnages politiques jouent sur la dérision et visent une distanciation critique ou philosophique, de nombreuses saynètes sont pétillantes de vie, vécue ou rêvée, toujours suivant les frémissements des fleurs-personnages. En effet, «Kalb Nakhla de l'Oasis Tozeur », «ce cœur de palmier» (41) qu'on connaît principalement sous le nom de Jasmin demeure une force constante dans les poétiques de relation que noue Lotus avec le monde. 


\section{Hymne à l'AmiAmour}

Ces poétiques de relations se manifestent dans les trajectoires et les transplantations symboliques de Lotus et de Jasmin. Elles traversent les cultures de l'Asie, de l'Afrique, de l'Europe et de l'Amérique en terme d'allers-retours multiples, plus souvent triangulaires que binaires (Laos-Thaïlande-France, Laos-France-Tunisie, Asie-Europe-Afrique, Asie-AfriqueAmérique). «Au pays du Lys », la réfugiée DorBoa et son voisin l'immigré Jasmin se sont donc liés d'une profonde amitié, «sans source irriguant l'affectif», et le narrateur en décrit l'originalité :

Chacun fait un pas et revient

Au miracle de l'AmiAmour inventif

Mais qu'est donc cette fleur exotique?

Un bien-être mariant amour et amitié

Pas très connu de grand monde et

Quand il est découvert joie/harmonie

Personne ne s'en rassasie! (122-23)

Cette amitié a son propre caractère. Si chaque personne est unique au monde, l'étoile qui en naît ne peut qu'être unique : mais, comme la descendance, elle porte les gènes de deux êtres qui obéissent à un même idéal porteur d'infini. Ici, elle ne tendra au jugement ni ne s'imposera, se découvrant et s'affinant au fil du temps, nourrie des expériences vécues. L’amour humain dépasse les frontières, et chacun le partage et le «subit sans en connaître l'habit ou l'acabit » (123). En effet, cet Ami/Amour porte remède aux blessures, aux regrets, et il ne connaît pas la haine ; sa main est tendue aux vulnérables; il répand le «Muguet de Paix » et le bonheur. Ainsi dira Lotus :

Son labeur consiste à cultiver les fleurs de toutes les couleurs

Candide et passionnée... non pour les butiner

Mais pour transporter leurs bonnes humeurs

Aux personnes de grandes nécessités ! (131)

Cet Ami/Amour s'appuie sur un humble substrat philosophique dans lequel s'enracine la spiritualité de Lotus ou la laïcité pratique de Jasmin, la foi chrétienne comme la foi islamique. Si les termes ne sont pas contradictoires, on dirait que Lotus est une passionnée tranquille, car elle « ne connaît... ni marge, ni centre, ni entre-deux ! »(132). Sa sagesse se confond avec son altruisme, et son bénévolat l'épanouit intérieurement. C'est une constante humaine : le don enrichit le donateur. 
Aux dernières saisons de son fleurir...

Lotus passe-partout

Vit au jour le jour sans projet de bâtir...

...ou de démolir

Elle se laisse vivre en routines habituelles...

...et ça suffit!

DorBoa se sent bien dans ses feuilles lotusiennes

En compagnie des [sic] toutes les fleurs d'Asie...

Elle rayonne

Son Jasmin reste son préféré

...pour le rire qui les façonne ! (132)

Malgré les enfarges politiques et la marginalisation qui en découle, DorBoa est demeurée ferme pour affronter toutes les difficultés de la vie quotidienne, car elle vivait de sa paix intérieure. Elle a dû pleurer, mais elle a été capable de rire aussi avec les siens et les autres. Preuve que l'Ami/Amour, sa force, était là, tout près, dans son cœur. Et tout devient facile avec lui.

Dans Bangkok Blues, l'intensité de la relation entre la Thaïlandaise Koï et le multiculturel Virgulius demeure comme en pénombre, les mots ne savent traduire l'oraison amoureuse ni l'effet désiré. C'est le toucher, « le dessin de trois petits cœurs qui se suivent en pas de danse » tracé sur son corps, qui anime son être et trace sa voie, comme celle du narrateur et de l'auteur : «Il recueille l'offrande mystérieuse pour y consteller la galaxie des esprits et émerge, archiviste des consciences des peuples migrateurs »(151). Au départ de son amant, Koï lui offre une orchidée et disparaît aussitôt (156). Le don de l'orchidée reste inexpliqué, et il fait partie de l'indicible de leur amour. Mais, voilà que cette fleur réapparaît dans La réfugiée, sous le nom de Dor Eung/Orchidée, « amie pour la vie » de DorBoa/Lotus, cette fois-ci comme symbole et grâce de l'AmiAmour.

L'hymne à 1'AmiAmour est révélateur de la démarche créatrice de Bouraoui qui tient à se rendre disponible, perméable, sensible à l'interaction infinie des êtres sous le prisme des diversités culturelles. La narratoème est à la fois source d'interrogation des altérités et souffle poétique de la nomandanse. La réfugiée met en scène plus qu'elle ne représente les interrogations que se posent le narrateur et les personnages sur la difficile cohabitation des communautés culturelles au sein de la nation. Ainsi, on interpelle le lecteur à découvrir et à participer à la nomadanse, c'est-à-dire à la connaissance et à la prise de conscience des altérités qui font vibrer le monde en perpétuel mouvement. 
La pratique d'une écriture si abondante et engagée crée une pépinière à ciel ouvert pour exprimer les consciences et les circonstances diverses de personnages aux prises avec la vie et la mort, dans la lumière ou la pénombre, pour confidences ou «verbophages ». La vie de Lotus dessinée en médaillons comme fleur transplantée, comme réfugiée politique, comme être minoritaire à respecter, c'est un talisman inspirant et un sourire en coin sur la route cahoteuse de l'émigressence. Elle offre un rayon d'optimisme, une lueur d'espoir sur une question incontournable de la modernité, autrement plus « engageante » que la mondialisation des marchés, car elle éveille le sentiment intense d'une corrélation essentielle qui unit les êtres et qui les fait vibrer au gré de leurs différences et de leurs sensibilités : un véritable hymne à l’AmiAmour,

\section{Bibliographie}

Bouraoui, Hédi. Émigressence. Ottawa : Vermillon, 1992.

---. Bangkok Blues. Ottawa : Vermillon, 1994.

---. Transvivance. Rennes : Aussant, 1996.

---. Transpoétique. Éloge du nomadisme. Montréal : Mémoire d'encrier, 2005.

---. La réfugiée (Lotus au pays du Lys). Toronto : CMC, 2012.

---. Mutante, la poésie. Toronto : CMC, 2015.

---. NomadiVivance I. Narratoème. Toronto : CMC, 2016.

Charpentier, Georges. «Des parcours littéraires en mosaïques ». Revue indépendante 338-238 (2013) : 18-21.

Claudel, Paul. Cent phrases pour éventail. Paris : Gallimard, [1942] 1996.

Collani, Tania. «Du seuil fragile qui sépare la prose de la poésie - un aperçu des tendances au $\mathrm{XX}^{\mathrm{e}}$ siècle» Acta fabula 11-2, Notes de lecture, février 2010. http://www.fabula.org/revue/document5507.php

Combe, Dominique. «Poésie et récit: le partage rhétorique d'Yves Bonnefoy ». L'information gramaticale 22-22 (1984) : 23-28.

http://www.persee.fr/web/revues/home/prescript/article/igram_0222838_1984_num_22_1_2242

Cotnam, Jacques, dir. Hédi Bouraoui, iconoclaste et chantre du transculturel. Hearst: Nordir, 1996. 
Déjeux, Jean. « Vésuviade ». IBLA 140 (1977) : 338.

Faïgou, Nafée Nelly. «Bouraoui, Hédi. La réfugiée (Lotus au pays du Lys). Toronto : CMC, 2012. » Voix plurielles 10-1 (2013) : 181-182.

Friedenkraft, Georges. «Ming et Mélusine». Images d'Asie et de femmes. www.adamantane.net/auteurs_edites/georges_friedenkraft

La Fontaine, Jean de. « Le loup et l'agneau ». Fables, Livre I, 10 : http://www.la-fontaine-chthierry.net/louagneau.htm.

Levin, J. R. « On Functions of Pictures in Prose ». Neuropsychological and Cognitive Processes in Reading. Dir. F.J. Pirozzolo et M. C. Wittrock. New York : Academic P, 1981. 203228.

Roumain, Jacques. Gouverneurs de la rosée. Port-au-Prince : Imprimerie de l'État, 1944.

Sabiston, Elizabeth. The Muse Strikes Back. Sudbury : Human Sciences Monograph Series 9 (2005).

Saint-Exupéry, Antoine de. Le petit prince. New York: Reynal et Hitchcock, 1943. http://www.cmls.polytechnique.fr/perso/tringali/documents/st_exupery_le_petit_prince.p $\underline{\text { df }}$

Tcheho, Isaac-Célestin. «La densité poétique de Bangkok blues, récit de voyage par Hédi Bouraoui. » Le discours de voyages: Afrique, Antilles. Dir. Romuald-Blaise Fonkoua. Paris : Karthala, 1998. 183-191.

\footnotetext{
NOTES

${ }^{1}$ Cette expression paraît en caractère gras dans La réfugiée.

${ }^{2}$ Clin d'œil au roman classique de Jacques Roumain, Gouverneurs de la rosée.

${ }^{3} \mathrm{Au}$ sujet de sa maîtrise du français, il est à noter que DorBoa a une prononciation des voyelles et des consonnes françaises fortement marquée par le lao : annuner $=$ annuler; montaille $=$ montagne; chep $=$ chef; champaille $=$ champagne; Espaille = Espagne (20), tantôt source charme, tantôt source de drame selon les péripéties de son vécu.

${ }^{4}$ Le personnage de Jasmin se rapproche, par son origine tunisienne et son parcours en France et au Canada, de celui de l'écrivain lui-même, Hédi Bouraoui. Il possède également son esprit critique, motivé par son sens de la justice, sa bienveillance à l'égard des autres et son souci du dialogue d'ouverture (86).

${ }^{5}$ Couverture dessinée par Micheline Montgomery. Illustrations internes de Martine Lamy, Sophie Olicki, Ody Saban, Claudine Goux, Gérard Sendry, Pierre Silvain, Laudine Jacobée, Adam Nidzgorski, Stoimen Stoilov.
} 